\title{
Elective and emergency laparoscopic cholecystectomy in the elderly: early or delayed approach
}

\author{
A Ferrarese*, V Martino, M Nano \\ From XXIII Annual Meeting of the Italian Society of Geriatric Surgery \\ Lecce, Italy. 2-4 December 2010
}

\section{Background}

Comparing outcomes of the first Division of Abdominal Surgery of the Saint Louis Hospital of Orbassano (Turin) with the literature, regarding timing and technique of early or delayed laparoscopic cholecystectomy in the management of acute cholecystitis in elderly patients.

\section{Materials and methods}

From January 2005 to December 2009 114 laparoscopic cholecystectomy in the elderly were performed in our surgical division: 67 for gallbladder stones and 47 for acute cholecystitis.

The diagnosis of cholecystitis and gallbladder stones was based on general condition, physical examination, laboratory, radiological findings and sepsis score. For the study we've also considered: total hospital stay, timing after and before the operation, kind and duration of operation, conversion to the open procedure, drain and final pathological results.

From this study 29 patients were excluded (17 for choledocolytiasis associated and 12 for hospitalisation > 20 days). We hadn't excluded patients ASA III and ASA IV: in these patients (27.4 \%, 17 ASA III and 4 ASA IV) abdominal pressure not superior of $10 \mathrm{mmHg}$ was used [1].

Elderly patients included in the study were $85(49 \mathrm{M}$, 36 F). Ordinary Cholecystectomy were peformed in 45 cases and Emergency Cholecystectomy in 40 cases. This last group was further divided into two groups [2-4]: DEA Early, E-DLC, (31 patients operated on within 72

\footnotetext{
* Correspondence: alessia.ferrarese@gmail.com Department of Clinical and Biologic Science, School of Medicine and Surgery "S. Luigi Gonzaga", AOU San Luigi Gonzaga, Orbassano, Turin - SCDU General Surgery, University of Turin, Italy
}

hours from onset of symptoms) and DEA Delayed, DDLC, (9 patients operated on after 72 hours to 9 days from onset of symptoms).

We've also considered the operating team (Table 1) that performed the operation because the first operator's experience was considered as an important factor in order to evaluate our results [5-11].

\section{Results}

The comparison between elective and emergency operations showed that drain placement and post operation hospital stay were found statistically significant in the emergency group (Table 2). There weren't any differences regarding team evaluation (Table 3). Concerning the analysis of the E-DLC and D-DLC groups there aren't any statistical differences (Table 4).

\section{Table 1 Definitions of equipes.}

\begin{tabular}{ll}
\hline Team 1 & $\begin{array}{l}\text { More than } 100 \text { laparoscopic cholecistectomy and more than } \\
100 \text { other laparoscopic operations. }\end{array}$ \\
Team 2 & $\begin{array}{l}\text { Less than } 100 \text { laparoscopic cholecistectomy and less than } 100 \\
\text { other laparoscopic operations. }\end{array}$ \\
Team 3 Surgeons in learning curve progression or Resident with expert \\
Surgeon supervisor
\end{tabular}

Table 2 Ord/DEA.

\begin{tabular}{cccc}
\hline & OC & DC & P Value \\
\hline Operation time (min) & $75,5(40-220)$ & $90(28-200)$ & 0.1874 \\
PO hospital stay (days) & $2(1-10)$ & $3(2-12)$ & 0.002313 \\
Conversion rate & $6.7 \%$ & $2 \%$ & 0.3869 \\
Complications & $8.5 \%$ & $2 \%$ & 0.2352 \\
Drains & $16.7 \%$ & $51 \%$ & 0.0003 \\
Associated operations & $13.3 \%$ & $12.8 \%$ & 0.998 \\
Cancer & $3 \%$ & 0 & - \\
\hline
\end{tabular}


Table 3 Equipes.

\begin{tabular}{cccc}
\hline Variabile & E1-E2 & E1-E3 & E2-E3 \\
\hline Operation time (min) & 0.6936 & 0.6089 & 0.2759 \\
PO hospital stay (days) & 0.3159 & 0.02131 & 0.09583 \\
Total hospital stay & 0.9362 & 0.004337 & 0.004981 \\
Conversion rate & 0.1553 & 0.6677 & 0.3896 \\
Complications & 0.3823 & 0.998 & 0.998 \\
\hline
\end{tabular}

Table 4 E-DLC/D-DLC.

\begin{tabular}{cccc}
\hline Parameter & ELC & DLC & P Value \\
\hline WBC & $11.05(3.73-28.8)$ & $9.05(2.23-15.6)$ & 0.03264 \\
PCR & $1.39(0.04-45)$ & $0.66(0.08-23.23)$ & 0.1672 \\
Temperature & $14 \%$ & $2(7 \%)$ & 0.5281 \\
Thickened wall & $57.4 \%$ & $13(48 \%)$ & 0.4 \\
Pericholecystic fluid & $17 \%$ & $2(7.4 \%)$ & 0.25 \\
Distended gallbladder & $43.4 \%$ & $12(44.4 \%)$ & 0.998 \\
Operation time (min) & $90(36-330)$ & $85(28-195)$ & 0.1554 \\
PO hospital stay (days) & $3(2-15)$ & $3(2-8)$ & 0.6551 \\
Total hospital stay & $4(2-16)$ & $10(4-16)$ & $\mathrm{p}<0,01$ \\
Tasso di conversione & $5 \%$ & $0 \%$ & 0.59 \\
Complications & $5 \%$ & $0 \%$ & 0.59 \\
Drains & $36 \%$ & $26 \%$ & 0.3752 \\
Operations associated & $8 \%$ & $15 \%$ & 0.2353 \\
Cancer & $1.6 \%$ & $0 \%$ & 0.998 \\
\hline
\end{tabular}

\section{Conclusions}

In contrast with other authors [12,13], laparoscopic cholecystectomy in our elderly patients, when performed with an adequate technique, represents a safe procedure to treat all cases of acute cholecystitis in an emergency setting [14-22]. Our technique represents a standardized surgical strategy to approach acute cholecystitis and cholelytiasis in the elderly in a safe, effective and reproducible manner.

\section{Published: 24 August 2011}

\section{References}

1. Catani M, Modini C: Laparoscopic cholecystectomy in acute cholecystitis: a proposal of safe and effective technique. Hepatogastroenterology 2007, 54(80):2186-91.

2. Chung-Mau Lo, Chi-Leung Liu, Sheung-Tat Fan, Edward C S Lai, John Wong: Prospective Randomized Study of Early Versus Delayed Laparoscopic Cholecystectomy for Acute Cholecystitis. Ann Surg 227(4):461-467.

3. Litwin DE, Cahan MA: Laparoscopic cholecystectomy. Surg Clin North Am 2008, 88(6):1295-313.

4. Wilson E, Gurusamy K, Gluud C, Davidson BR: Cost-utility and value-ofinformation analysis of early versus delayed laparoscopic cholecystectomy for acute cholecystitis. Br J Surg 2010, 97:210-219.

5. Dàvila D, Manzanares C, Picho -ML, Albors P, Cardenas F, Fuster E: Experience in the treatment (early vs. delayed) of acute cholecystitis via laparoscopy. Cirugia Espanola 1999, 66:233.

6. Bohacek L, MD David, E Pace: Advanced laparoscopic training and outcomes in laparoscopic cholecystectomy. Can J Surg 2009, 52:4.
7. Ballantyne GH, Ewing D, Capella RF: The learning curve measured by operating times for laparoscopic and open gastric bypass: roles of surgeon's experience, institutional experience, body mass index and fellowship training. Obes Surg 2005, 15:172-82.

8. Gill J, Booth MI, Stratford J, Dehn TC: The extended learning curve for laparoscopic fundoplication: a cohort analysis of 400 consecutive cases J Gastrointest Surg 2007, 11:487-92.

9. Avital S, Hermon H, Greenberg R, Karin E, Skornick Y: Learning curve in laparoscopic colorectal surgery: our first 100 patients. Isr Med Assoc J 2006, 8(10):683-6.

10. Soot SJ, Eshraghi N, Farahmand M, Sheppard BC, Deveney CW: Transition from open to laparoscopic fundoplication: the learning curve. Arch Surg 1999, 134(3):278-81.

11. Li GX, Yan HT, Yu J, Lei ST, Xue Q, Cheng X: Learning curve of laparoscopic resection for rectal cancer. Nan Fang Yi Ke Da Xue Xue Bao 2006, 26(4):535-8.

12. Kauvar DS, Brown BD, Braswell AW, Harnisch MJ: Laparoscopic cholecystectomy in the elderly: increased operative complications and conversions to laparotomy. Laparoendosc Adv Surg Tech A 2005, 15(4):379-82.

13. Moyson J, Thill V, Simoens Ch, Smets D, Debergh N, Mendes da Costa P: Laparoscopic cholecystectomy for acute cholecystitis in the elderly: a retrospective study of 100 patients. Hepatogastroenterology 2008, 55(88):1975-80.

14. Lau H, Lo CY, Patil NG, Yuen WK: Early versus delayed-interval laparoscopic cholecystectomy for acute cholecystitis: a metaanalysis. Surg Endosc 2006, 20(1):82-7.

15. Johansson M, Thune A, Blomqvist A, Nelvin L, Lundell L: Management of acute cholecystitis in the laparoscopic era: results of a prospective, randomized clinical trial. J Gastrointest Surg 2003, 7:642-645.

16. Lo C, Liu C, Fan ST, Lai EC, Wong J: Prospective randomized study of early versus delayed laparoscopic cholecystectomy for acute cholecystitis. Ann Surg 1998, 227:461-467.

17. Polychronidis A, Botaitis S, Tsaroucha A, Tripsianis G, Bounovas A, Pitiakoudis M, Simopoulos C: Laparoscopic cholecystectomy in elderly patients. J Gastrointestin Liver Dis 2008, 17(3):309-13.

18. Kirshtein B, Bayme M, Bolotin A, Mizrahi S, Lantsberg L: Laparoscopic cholecystectomy for acute cholecystitis in the elderly: is it safe? Surg Laparosc Endosc Percutan Tech 2008, 18(4):334-9.

19. Majeski J: Laparoscopic cholecystectomy in geriatric patients. Am J Surg 2004, 187(6):747-50.

20. Stanisić V, Bakić M, Magdelinić M, Kolasinac H, Miladinović M: Laparoscopic cholecystectomy in elderly patients. Acta Chir lugos/ 2009, 56(2):87-91.

21. Tambyraja AL, Kumar S, Nixon SJ: Outcome of laparoscopic cholecystectomy in patients 80 years and older. World J Surg 2004, 28(8):745-8.

22. Behrman SW, Melvin WS, Babb ME, Johnson J, Ellison EC: Laparoscopic cholecystectomy in the geriatric population. Am Surg 1996, 62(5):386-90.

doi:10.1186/1471-2318-11-S1-A14

Cite this article as: Ferrarese et al:: Elective and emergency laparoscopic cholecystectomy in the elderly: early or delayed approach. BMC

Geriatrics 2011 11(Suppl 1):A14.

\section{Submit your next manuscript to BioMed Central and take full advantage of:}

- Convenient online submission

- Thorough peer review

- No space constraints or color figure charges

- Immediate publication on acceptance

- Inclusion in PubMed, CAS, Scopus and Google Scholar

- Research which is freely available for redistribution 